\title{
Minimum Total Dominating Energy of Some Standard Graphs
}

\author{
K. Malathy, S. Meenakshi
}

\begin{abstract}
Let $G$ be a simple graph with vertex set $V(G)$ and edge set $E(G)$. A set $S$ of vertices in a graph $G(V, E)$ is called a total dominating set if every vertex $v \in V$ is adjacent to an element of $S$. The minimum cardinality of a total dominating set of $G$ is called the total domination number of $G$ which is denoted by $\gamma_{t}(G)$. The energy of the graph is defined as the sum of the absolute values of the eigen values of the adjacency matrix. In this paper, we computed minimum total dominating energy of a Friendship Graph, Ladder Graph and Helm graph. The Minimum total dominating energy for bistar graphand sun graph is also determined.
\end{abstract}

Keywords: dominating set, minimum total dominating set, minimum total dominating matrix, minimum total dominating eigen values, minimum total dominating energy of a graph.

\section{INTRODUCTION}

In 1960, the mathematical study of Domination Theory in graphs was started.I.Gutman introduced the concept of energy of a graph in the year 1978. The roots go back to 1862 when C.F. De Jaenisch studied the problem of determining the minimum number of queens necessary to cover an $n \times n$ chess board in such way that every square is attacked by one of the queens.

The graph invariant is closely connected to a chemical quantity known as the total $\pi$ electron energy of conjugated hydrocarbon molecules. The study of GraphEnergy first arouses in the field of chemistry. Chemists used Huckel's method to approximate energies associated with $\pi$ - electron orbitals in a special class of molecules called conjugated hydrocarbons. Gutman first introduced the concept of 'energy of graph' for a simple graph. At first very few mathematicians seemed to be interested in this concept. However, over the years graph energy has become an interesting area of research for mathematicians and several variations have been introduced.

Let $\mathrm{G}$ be a graph with x-vertices and y-edges. Let $A=\left(a_{i j}\right)$ be the adjacency matrix of a graph. Let $\lambda_{1}, \lambda_{2}, \ldots ., \lambda_{n}$ be the eigenvalues of adjacency matrix of a graph $\mathrm{G}$. The values are in non-decreasing order, that is $\lambda_{1} \geq \lambda_{2} \geq \ldots . \geq \lambda_{n}$. Since $A(G)$ is real and symmetric, its eigenvalues are real number. The energy $E(G)$ of graph is defined as sum of the absolute values of its eigenvalues of graph $\mathrm{G}$,ie, $E(G)=$ $\sum_{i=1}^{n}\left|\lambda_{i}\right|$

\footnotetext{
Revised Manuscript Received on December 5, 2019.

K.Malathy, Research Scholar, Department of Mathematics, VISTAS, Chennai.

Dr. S. Meenakshi, Associate Professor, Department of Mathematics, VISTAS, Chennai
}

In this paper, we computed minimum total dominating energy of a Friendship Graph, Ladder Graph and Helm graph.The Minimum total dominating energy for bistar graphand sun graph is also determined.

\section{PRELIMINARIES}

\section{Definition 2.1Dominating set}

A dominating set for a graph $G=(V, E) \quad$ is a subset $\mathrm{D}$ of $\mathrm{V}$ such that every vertex not in $\mathrm{D}$ is adjacent to at least one member of D.The minimum cardinality of a dominating set of $G$ is called the domination number of $\mathrm{G}$ which is denoted by $\gamma(G)$. [2][5]

\section{Definition 2.2 Total Dominating set}

A set $\mathrm{S}$ of vertices in a graph $\mathrm{G}(\mathrm{V}, \mathrm{E})$ is called a total dominating set if every vertex $\mathrm{v} \in \mathrm{V}$ is adjacent to an element of $\mathrm{S}$. The minimum cardinality of a total dominating set of $\mathrm{G}$ is called the total domination number of $\mathrm{G}$ which is denoted by $\gamma_{\mathrm{t}}(\mathrm{G})$. [1]

\section{Definition 2.3 Energy}

Energy of a simple graph $G=(V, E)$ with adjacency matrix $A$ is defined as the sum of absolute values of eigen values of A denoted by $E(G)$. ie, $E(G)=\sum_{i=1}^{n}\left|\lambda_{i}\right|$ where $\lambda_{i}$ is an eigen values of $A, i=1,2, \ldots . n .[3][4]$

\section{Definition 2.4 Minimum Total Dominating Energy}

Let $\mathrm{G}$ be a simple graph of order $n$ with vertex set $V=$ $\left\{v_{1}, v_{2}, \ldots . v_{n}\right\}$ and the edge set E. A vertex set $S$ in $G$ is a total dominating setif every vertex $\mathrm{v} \in \mathrm{V}$ is adjacent to an element of $\mathrm{S}$

The total dominating number is the minimum cardinality taken over all theminimal total dominating sets of $\mathrm{G}$. LetTDSbe the minimum total dominating set of graph $\mathrm{G}$. The minimum total dominating matrix of $G$ isn $\times n$ matrix $\mathrm{A}_{\mathrm{TD}}(\mathrm{G})=\left(\mathrm{a}_{\mathrm{ij}}\right)$ where,

$$
A_{T D}(G)=\left\{\begin{array}{lr}
1 \text { if } v_{i} \text { and } v_{j} \text { are adjacent } \\
1 & \text { if } i=j, v_{i} \in \text { TDS } \\
0 & \text { otherwise }
\end{array}\right.
$$

The characteristic polynomial of $A_{T D}(G)$ is denoted $\operatorname{byP}(G, \lambda)=\operatorname{det}\left(\lambda I-A_{\mathrm{TD}}(G)\right)$. The minimum total dominating eigen values of the graph $G$ are the eigen values of $A_{T D}(G)$.

Since $A_{T D}(G)$ is real and symmetric, the eigen values are $\lambda_{1}, \lambda_{2}, \ldots ., \lambda_{n}$ in decreasing order $\lambda_{1} \geq \lambda_{2} \geq, \ldots \geq$ $\lambda_{\mathrm{n}}$. The total dominating energy of $\mathrm{G}$ is defined as,

$\mathrm{E}_{\mathrm{TD}}(\mathrm{G})=\sum_{\mathrm{i}=1}^{\mathrm{n}}\left|\lambda_{\mathrm{i}}\right| \cdot[6][7]$ 


\section{Example:}

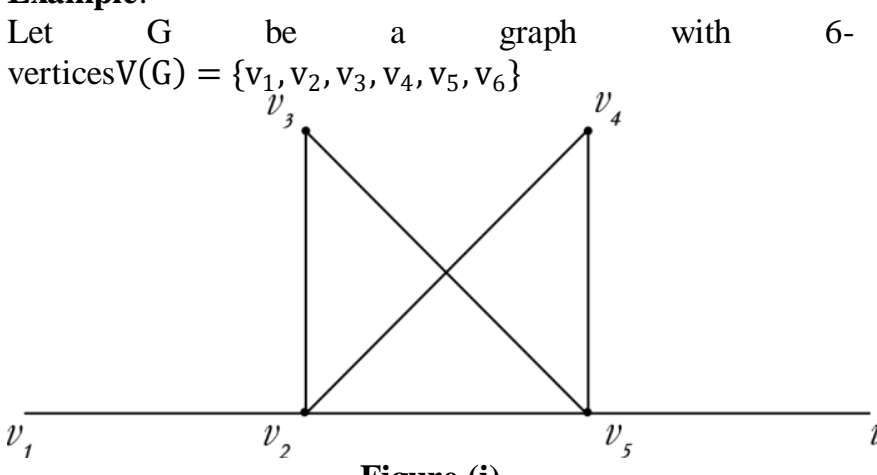

Figure (i)

The minimum total dominating set, TDS $=\left\{\mathrm{v}_{2}, \mathrm{v}_{5}\right\}$.

The minimum cardinality of a total dominating $\operatorname{set} \gamma_{\mathrm{t}}(\mathrm{G})=$ 2 .

The minimum total dominating matrix, $A_{T D}(G)=$

$\left(\begin{array}{llllll}0 & 1 & 0 & 0 & 0 & 0 \\ 1 & 1 & 1 & 1 & 1 & 0 \\ 0 & 1 & 0 & 0 & 1 & 0 \\ 0 & 1 & 0 & 0 & 1 & 0 \\ 0 & 1 & 1 & 1 & 1 & 1 \\ 0 & 0 & 0 & 0 & 1 & 0\end{array}\right)$

The Characteristic polynomial is $x^{6}-2 x^{5}-6 x^{4}+2 x^{3}+$ $5 \mathrm{x}^{2}+0 \mathrm{x}-0$

The eigen values are $\lambda_{1}=-1.4495 \lambda_{2}=-1.0000 \lambda_{3}=$ $-0.0000$

$\lambda_{4}=0.0000 \quad \lambda_{5}=1.0000 \lambda_{6}=3.4495$

Therefore, the minimum total dominating energy, $\mathrm{E}_{\mathrm{TD}}(\mathrm{G})=$ 6.899

\section{MINIMUM TOTAL DOMINATING ENERGY OF SOME STANDARD GRAPH}

\section{Definition 3.1 Ladder graph:}

The ladder graph $L_{n}$ is defined by $L_{n}=P_{n} \times K_{2}$ where $P_{n}$ is a path with $n$ vertices and

$\times$ denotes the Cartesian product and $K_{2}$ is a complete graph with two-vertices.

\section{Definition 3.2 Friendship graph:}

The friendship graph $F_{n}$ can be constructed by joining $n$ copies of the cycle graph $C_{n}$ with a common vertex

\section{Definition 3.3 Helm graph:}

The helm graph $H_{n}$ is the graph obtained from an nwheel graph by adjoining a pendant edge at each node of the cycle.

\section{Definition 3.4 Sun graph:}

The Sun graph of order 2nis cycle with edges terminating in a pendantvertex attached to each vertex and it is denoted by $\mathrm{SG}_{\mathrm{n}}$.

\section{Definition 3.5 Bistar graph:}

The graph $B_{n, n} n>2$ is a bistar obtained from two disjoint copies of $K_{1, n}$ by joining the centre vertices by an edge. It has $2 n+2$ vertices and $2 n+1$ edges.

\section{Theorem 3.1}

The minimum total dominating energy of Ladder graph $L_{n}$ is $\frac{1 \pm \sqrt{(1+8 n)}}{2}$.

\section{Proof}

Let $\mathrm{G}=\mathrm{L}_{\mathrm{n}}$ be a ladder graph on nvertices with

$$
\mathrm{V}(\mathrm{G})=\left\{\mathrm{u}_{\mathrm{i}}, \mathrm{v}_{\mathrm{i}}: 1 \leq \mathrm{i} \leq 1\right\} \text { and }
$$

$$
\mathrm{E}(\mathrm{G})=\left\{\mathrm{u}_{\mathrm{i}} \mathrm{u}_{\mathrm{i}+1}, \mathrm{v}_{\mathrm{i}} \mathrm{v}_{\mathrm{i}+1}: 1 \leq \mathrm{i} \leq \mathrm{n}-1\right\} \cup\left\{\mathrm{u}_{\mathrm{i}} \mathrm{v}_{\mathrm{i}}: 1 \leq \mathrm{i}\right.
$$

Consider the ladder graph $L_{n}$ with vertex set $\left\{v_{1}, v_{2}, v_{3}, v_{4} \ldots \ldots v_{n}, v_{n-1}\right\}$

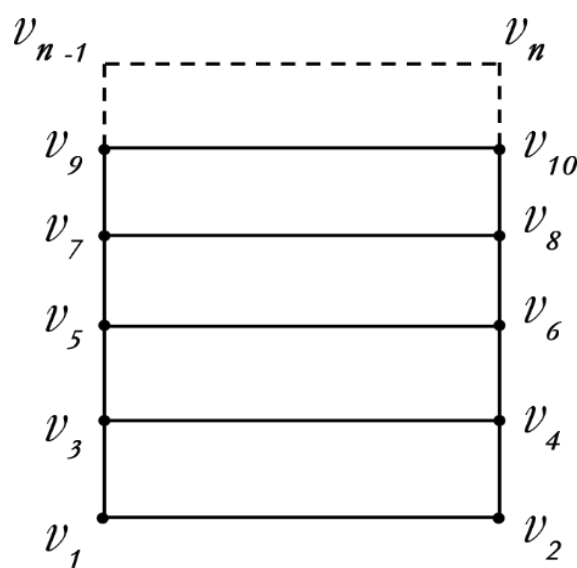

Figure (i) Ladder Graph

The minimum total dominating set, $T D S=\left\{v_{1}, v_{3}, v_{5}, v_{7} \ldots \ldots v_{n-1}\right\}$

The minimum cardinality of a total dominating $\operatorname{set} \gamma_{t}(G)=$ $n / 2$.

The total dominating matrix is given by

$$
A_{T D}\left(L_{n}\right)=\left(\begin{array}{cccccc}
1 & 1 & 1 & \ldots & 0 & 0 \\
1 & 0 & 0 & \ldots & 0 & 0 \\
1 & 0 & 0 & \ldots & 0 & 0 \\
\vdots & \vdots & \vdots & \ldots & \vdots & \vdots \\
0 & 0 & 0 & \ldots & 1 & 1 \\
0 & 0 & 0 & \ldots & 1 & 0
\end{array}\right)
$$

The Characteristic polynomial

$\operatorname{det}\left(\lambda I-A_{T D}\left(L_{n}\right)\right)=\left|\begin{array}{cccccc}\lambda-1 & -1 & -1 & \ldots & 0 & 0 \\ -1 & \lambda & 0 & \ldots & 0 & 0 \\ -1 & 0 & \lambda & \ldots & 0 & 0 \\ \vdots & \vdots & \vdots & \ddots & \vdots & \vdots \\ 0 & 0 & 0 & \ldots & \lambda-1 & -1 \\ 0 & 0 & 0 & \ldots & -1 & \lambda\end{array}\right|$

The characteristic equation is $\lambda^{n-3}\left(\lambda^{3}-\lambda^{2}-2 n \lambda\right)=0$

The eigen values are, $\lambda=0(n-3)$ times, $\lambda=\frac{1 \pm \sqrt{(}(1+8 n)}{2}$

The minimum total dominating energy of Ladder graph $L_{n}$, $E_{T D}\left(L_{n}\right)=\frac{1 \pm \sqrt{ }(1+8 n)}{2}$

\section{Theorem 3.2}

The minimum total dominating energy of Fiendship graph $F_{n}$ is $1 \pm \sqrt{n-2}$.

\section{Proof}

Since the center vertex of Friendship graph are minimum dominating set of G.the minimum total dominating set depends upon the center vertex of $\mathrm{G}$, and some other vertex which is adjacent to center vertex.

Hence the minimum total dominating energy depends upon the center vertex and some other vertex which is adjacent to center vertex.

Consider the Friendship graph $F_{n}$ with vertex set $\left\{v_{1}, v_{2}, \ldots \ldots v_{n}\right\}$ 


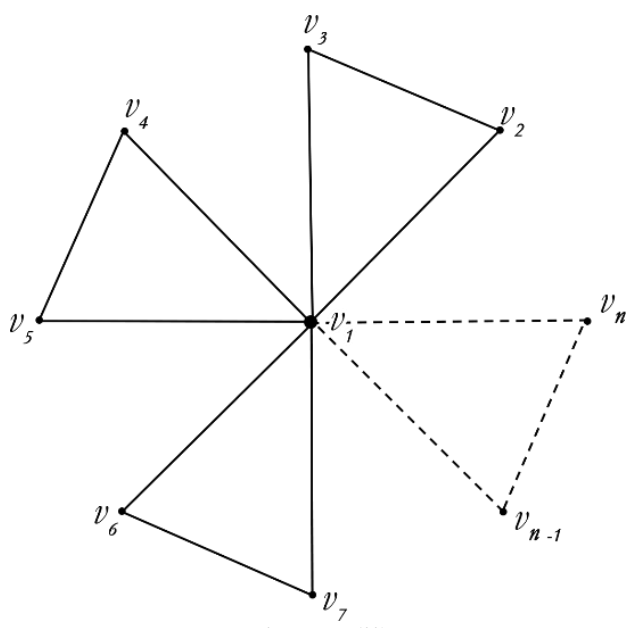

Figure (ii)

Friendship Graph

The minimum total dominating set,TDS $=\left\{v_{1}, v_{2}\right\}$

The minimum cardinality of a total dominating $\operatorname{set} \gamma_{t}(G)=$ 2 .

The total dominating matrix is given by

$$
A_{T D}\left(F_{n}\right)=\left(\begin{array}{cccccc}
1 & 1 & 1 & \ldots & 1 & 1 \\
1 & 1 & 1 & \ldots & 0 & 0 \\
1 & 1 & 0 & \ldots & 0 & 0 \\
\vdots & \vdots & \vdots & \ldots & \vdots & \vdots \\
1 & 0 & 0 & \ldots & 0 & 1 \\
1 & 0 & 0 & \ldots & 1 & 0
\end{array}\right)
$$

The Characteristic polynomial

$\operatorname{det}\left(\lambda I-A_{T D}\left(F_{n}\right)\right)=\left|\begin{array}{cccccc}\lambda-1 & -1 & -1 & \ldots & -1 & -1 \\ -1 & \lambda-1 & -1 & \ldots & 0 & 0 \\ -1 & -1 & \lambda & \ldots & 0 & 0 \\ \vdots & \vdots & \vdots & \ddots & \vdots & \vdots \\ -1 & 0 & 0 & \ldots & \lambda & -1 \\ -1 & 0 & 0 & \ldots & -1 & \lambda\end{array}\right|$ $R_{i} \rightarrow R_{i}-R_{4}, i=3,4,5 \ldots . n$

$$
\left|\begin{array}{cccccc}
\lambda-1 & -1 & -1 & \ldots & -1 & -1 \\
-1 & \lambda-1 & 0 & \ldots & 0 & 0 \\
0 & 0 & \lambda & \ldots & 0 & 0 \\
\vdots & \vdots & \vdots & \ddots & \vdots & \vdots \\
0 & 0 & 0 & \ldots & \lambda & -1 \\
0 & 0 & 0 & \ldots & -1 & \lambda
\end{array}\right|
$$

The characteristic equation is $\lambda^{n-3}\left(\lambda^{2}-2 \lambda+3-n\right)=0$ The eigen values are, $\lambda=0(n-3)$ times, $\quad \lambda=1 \pm$ $\sqrt{n-2}$

The minimum total dominating energy of Friendship graph $F_{n}, E_{T D}\left(F_{n}\right)=1 \pm \sqrt{n-2}$

\section{Theorem 3.3}

The minimum total dominating energy of Helm graph $H_{n}$ is $-n \pm \sqrt{\frac{n(n-1)}{2}}$.

\section{Proof}

Consider the Helm graph $H_{n}$ with vertex set $\left\{v_{1}, v_{2}, \ldots \ldots v_{n}\right\}$

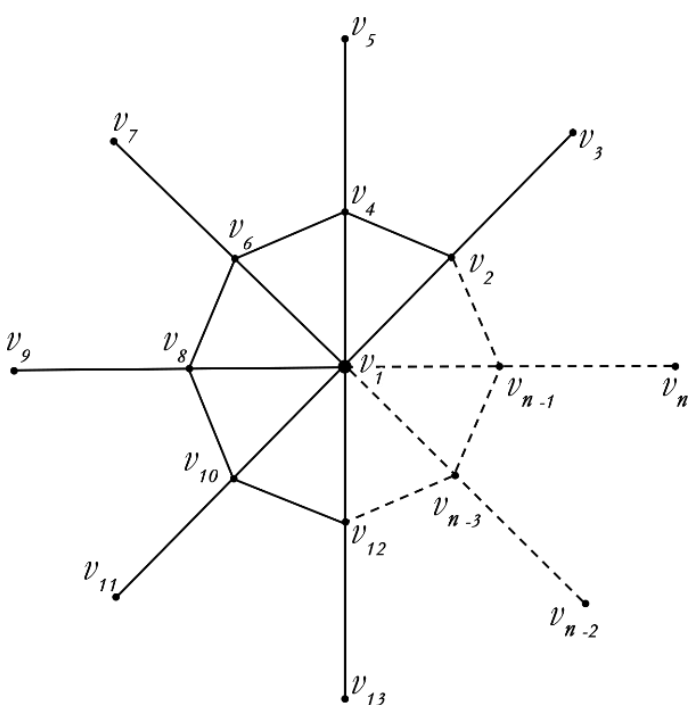

Figure (iii)

Helm Graph

The minimum total dominating set, $T D S=\left\{v_{1}, v_{2}, v_{4}, v_{6}, v_{8}, \ldots . v_{n-1}\right\}$

The minimum cardinality of a total dominating $\operatorname{set} \gamma_{t}(G)=$ $(n+1) / 2$.

The total dominating matrix is given by

$$
A_{T D}\left(H_{n}\right)=\left(\begin{array}{cccccc}
1 & 1 & 0 & \ldots & 1 & 0 \\
1 & 1 & 1 & \ldots & 0 & 0 \\
0 & 1 & 0 & \ldots & 0 & 0 \\
\vdots & \vdots & \vdots & \ldots & \vdots & \vdots \\
1 & 0 & 0 & \ldots & 1 & 1 \\
0 & 0 & 0 & \ldots & 1 & 0
\end{array}\right)
$$

The Characteristic polynomial

$$
\begin{aligned}
& \operatorname{det}\left(\lambda I-A_{T D}\left(H_{n}\right)\right) \\
& =\left|\begin{array}{cccccc}
\lambda-1 & -1 & 0 & \ldots & -1 & 0 \\
-1 & \lambda-1 & -1 & \ldots & 0 & 0 \\
0 & -1 & \lambda & \ldots & 0 & 0 \\
\vdots & \vdots & \vdots & \ddots & \vdots & \vdots \\
-1 & 0 & 0 & \ldots & \lambda-1 & -1 \\
0 & 0 & 0 & \ldots & -1 & \lambda
\end{array}\right|
\end{aligned}
$$

The characteristic equation is $\lambda^{n-3}\left(\lambda^{2}-(2 n) \lambda+n\right)=0$

The eigen values are, $\lambda=0(n-3)$ times, $\quad \lambda=-n \pm$ $\sqrt{\frac{n(n-1)}{2}}$

The minimum total dominating energy of Helm graph $H_{n}$, $E_{T D}\left(H_{n}\right)=-n \pm \sqrt{\frac{n(n-1)}{2}}$

\section{Theorem 3.4}

The minimum total dominating energy of Sun graphSG $G_{n}$ is $1 \pm \sqrt{n+3}$. 


\section{Proof}

Consider the Sun graph $S G_{n}$ with vertex set $\left\{v_{1}, v_{2}, \ldots \ldots v_{n}\right\}$

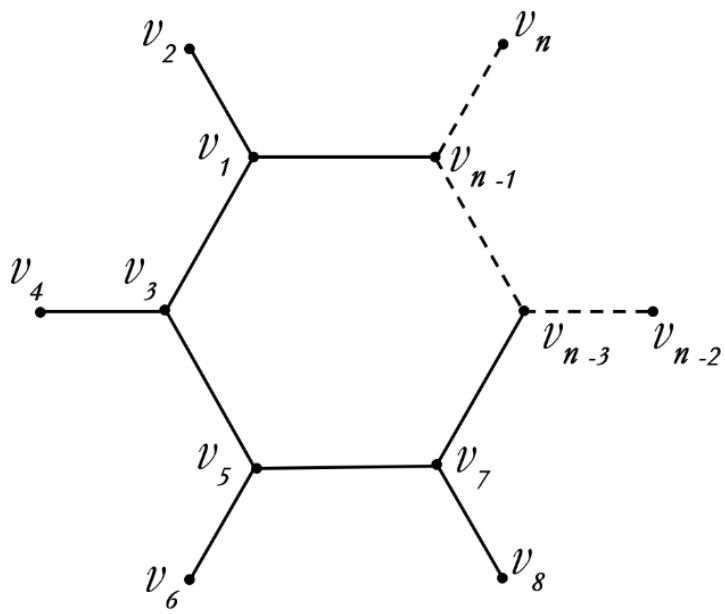

Figure (iv)

Sun Graph

The minimum total
set, $T D S=\left\{v_{1}, v_{3}, v_{5}, v_{7}, v_{9}, \ldots . v_{n-1}\right\}$

dominating

The minimum cardinality of a total dominating $\operatorname{set}_{\mathrm{t}}(\mathrm{G})=$ $\mathrm{n} / 2$.

The total dominating matrix is given by

$$
\mathrm{A}_{\mathrm{TD}}\left(\mathrm{SG}_{\mathrm{n}}\right)=\left(\begin{array}{cccccc}
1 & 1 & 1 & \ldots & 1 & 0 \\
1 & 0 & 0 & \ldots & 0 & 0 \\
1 & 0 & 1 & \ldots & 0 & 0 \\
\vdots & \vdots & \vdots & \ldots & \vdots & \vdots \\
1 & 0 & 0 & \ldots & 1 & 1 \\
0 & 0 & 0 & \ldots & 1 & 0
\end{array}\right)
$$

The Characteristic polynomial

$$
\operatorname{det}\left(\lambda \mathrm{I}-\mathrm{A}_{\mathrm{TD}}\left(\mathrm{SG}_{\mathrm{n}}\right)\right)
$$

$$
=\left|\begin{array}{cccccc}
\lambda-1 & -1 & -1 & \ldots & -1 & 0 \\
-1 & \lambda & 0 & \ldots & 0 & 0 \\
-1 & 0 & \lambda-1 & \ldots & 0 & 0 \\
\vdots & \vdots & \vdots & \ddots & \vdots & \vdots \\
-1 & 0 & 0 & \ldots & \lambda-1 & -1 \\
0 & 0 & 0 & \ldots & -1 & \lambda
\end{array}\right|
$$

$R_{i} \rightarrow R_{i}-R_{4}, i=5,6,7 \ldots . n$

$$
\left|\begin{array}{cccccc}
\lambda-1 & -1 & -1 & \ldots & -1 & 0 \\
-1 & \lambda & 0 & \ldots & 0 & 0 \\
-1 & 0 & \lambda-1 & \ldots & 0 & 0 \\
\vdots & \vdots & \vdots & \ddots & \vdots & \vdots \\
0 & 0 & 0 & \ldots & \lambda & 0 \\
0 & 0 & 0 & \ldots & 0 & \lambda
\end{array}\right|
$$

The characteristic equation is $\lambda^{n-3}\left(\lambda^{2}-\lambda-(3+n)\right)=0$ The eigen values are, $\lambda=0(n-3)$ times, $\lambda=1 \pm \sqrt{n+3}$ The minimum total dominating energy of star graph $S_{n}$, $\mathrm{E}_{\mathrm{TD}}\left(\mathrm{SG}_{\mathrm{n}}\right)=1 \pm \sqrt{\mathrm{n}+3}$

\section{Theorem 3.5}

The minimum total dominating energy of Bistar graphB $\mathrm{B}_{\mathrm{n}, \mathrm{n}}$ is $(2 n-5)+\sqrt{n^{2}-4 n+8}$.

\section{Proof}

Consider the Bistar graph $B_{n, n}$ with vertex set $\left\{\mathrm{v}_{1}, \mathrm{u}_{1}, \mathrm{v}_{2}, \mathrm{u}_{2} \ldots \ldots \mathrm{v}_{\mathrm{n}}, \mathrm{u}_{\mathrm{n}}\right\}$
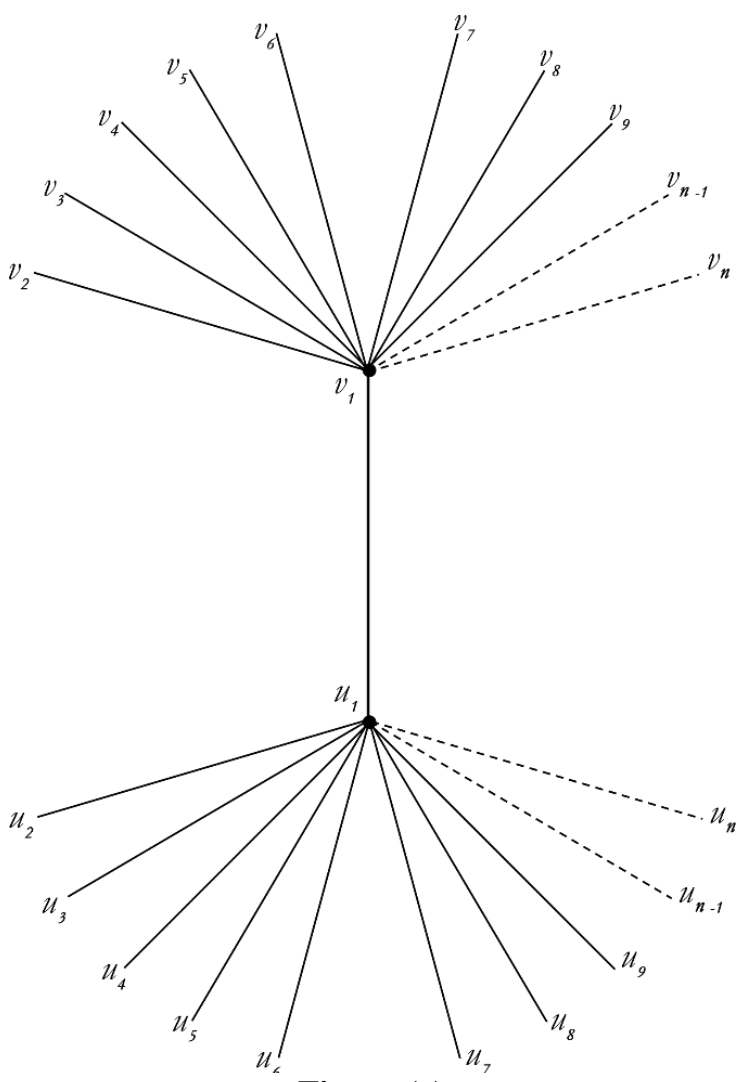

Figure (v)

Bistar Graph

The minimum total dominating set, TDS $=\left\{\mathrm{v}_{1}, \mathrm{u}_{1}\right\}$

The minimum cardinality of a total dominating $\operatorname{set} \gamma_{\mathrm{t}}(\mathrm{G})=$ 2.

The total dominating matrix is given by

$$
\mathrm{A}_{\mathrm{TD}}\left(\mathrm{B}_{\mathrm{n}, \mathrm{n}}\right)=\left(\begin{array}{cccccc}
1 & 1 & 1 & \ldots & 1 & 0 \\
1 & 1 & 0 & \ldots & 0 & 1 \\
1 & 0 & 0 & \ldots & 0 & 0 \\
\vdots & \vdots & \vdots & \ldots & \vdots & \vdots \\
1 & 0 & 0 & \ldots & 0 & 0 \\
0 & 1 & 0 & \ldots & 1 & 0
\end{array}\right)
$$

The Characteristic polynomial

$$
\begin{aligned}
\operatorname{det}\left(\lambda I-A_{\mathrm{TD}}\left(\mathrm{B}_{\mathrm{n}, \mathrm{n}}\right)\right) & =\left|\begin{array}{cccccc}
\lambda-1 & -1 & -1 & \ldots & -1 & 0 \\
-1 & \lambda-1 & 0 & \ldots & 0 & -1 \\
-1 & 0 & 0 & \ldots & 0 & 0 \\
\vdots & \vdots & \vdots & \ddots & \vdots & \vdots \\
-1 & 0 & 0 & \ldots & \lambda & 0 \\
0 & -1 & 0 & \ldots & -1 & \lambda
\end{array}\right|
\end{aligned}
$$

The characteristic equation is $(\lambda+1)^{n-3}\left(\lambda^{2}-(n-2) \lambda-\right.$ $1=0$

The eigen values are, $\lambda=-1(n-3)$ times, $\lambda=(n-2)+$ $\sqrt{n^{2}-4 n+8}$

The minimum total dominating energy of bistar graph $B_{n, n}$,

$\mathrm{E}_{\mathrm{TD}}\left(\mathrm{B}_{\mathrm{n}, \mathrm{n}}\right)=|-1|(\mathrm{n}-3)+\left\{(\mathrm{n}-2)+\sqrt{\mathrm{n}^{2}-4 \mathrm{n}+8}\right\}$ $=(n-3)+\{(n-2)+$ $\left.\sqrt{n^{2}-4 n+8}\right\}$ $=(2 n-5)+\sqrt{n^{2}-4 n+8}$ 


\section{CONCLUSION}

In this paper, the energy namely Minimum total dominating energy is defined and has beenfound forFriendship Graph, Ladder Graph and Helm graph.The Minimum total dominating energy for bistar graphand sun graph is also determined.For future research, the minimum inverse total dominating energy can be computed for some standard graphs.

\section{REFERENCES}

1. E.J. Cockayne, R.M. Dawes and S.T. Hedetniemi, Total dominations in graphs, Networks, 10 (1980), 211-219.

2. E.J. Cockayne, and S.T. Hedetniemi, Towards a theory of domination in graphs, Networks, (1977), pp. 247-261

3. I.Gutman, The energy of a graph, Ber. Math. Statist. Sket. for Schungsz, Graz, 103 (1978)1-22.

4. I.Gutman, The energy of a graph: Old and new results (Ed-s: A.Betten, A.Kohnert, R.Lau,A.Wassermann), Algebraic combinatorics and Applications, Sringer, berlin,96-211(2001).

5. V.R.Kulli, Theory of domination in graphs, Vishwa International Publications, Gulbarga, India, 2010

6. Meenakshi S. and S.Lavanya, A Survey on Energy of Graphs, Annals of pure and applied mathematics, Vol 8, No. 2, 2014, 183 191, ISSN:2279-087X(P). 17 December(2014)

7. M.R.RajeshKanna, B.N.Dharmndra and G.Sridhara, The Minimum dominating energy of a graph, International Journal of Pure and Applied Mathematics, 85 (4) (2013) 707-718.

\section{AUTHORS PROFILE}

K.Malathy, Research Scholar, Department of Mathematics, VISTAS, Chennai.

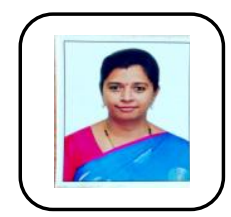

Dr. S. Meenakshi, Associate Professor, Department of Mathematics, VISTAS, Chennai 